\title{
The Maria Curie-Skłodowska University Botanical Garden in Lublin as a refuge of the moths (Lepidoptera: Heterocera) within the city
}

\author{
ŁUKASZ DAWIDOWICZ, ${ }^{1}$ HALINA KUCHARCZYK ${ }^{2}$ \\ Department of Zoology, Maria Curie-Skłodowska University, Akademicka 19, 20-033 Lublin, Poland \\ e-mail: mori666@o2.pl \\ e-mail: halina.kucharczyk@poczta.umcs.lublin.pl
}

Keywords biodiversity, urban fauna, faunistics, city, species composition, rare species, conservation

Abstract In 2012 and 2013, 418 species of moths at total were recorded in the Botanical Garden of the Maria Curie-Skłodowska University in Lublin. The list comprises 116 species of Noctuidae (26.4\% of the Polish fauna), 116 species of Geometridae (28.4\% of the Polish fauna) and 63 species of other Macrolepidoptera representatives (27.9\% of the Polish fauna). The remaining 123 species were represented by Microlepidoptera. Nearly $10 \%$ of the species were associated with wetland habitats, what constitutes a surprisingly large proportion in such an urbanised area. Comparing the obtained data with previous studies concerning Polish urban fauna of Lepidoptera, the moths assemblages in the Botanical Garden were the most similar to the one from the Natolin Forest Reserve which protects the legacy of Mazovian forests. Several recorded moths appertain to locally and rarely encountered species, as Stegania cararia, Melanthia procellata, Pasiphila chloerata, Eupithecia haworthiata, Horisme corticata, Xylomoia graminea, Polychrysia moneta. In the light of the conducted studies, the Botanical Garden in Lublin stands out as quite high biodiversity and can be regarded as a refuge for moths within the urban limits of Lublin.

\section{Ogród Botaniczny Uniwersytetu Marii Curie-Skłodowskiej w Lublinie} jako ostoja motyli nocnych (Lepidoptera: Heterocera) w mieście

Słowa kluczowe bioróżnorodność, fauna miejska, faunistyka, miasto, struktura gatunkowa, gatunki rzadkie, ochrona.

Streszczenie Na podstawie badań przeprowadzonych w latach 2012-2013 na terenie Ogrodu Botanicznego Uniwersytetu Marii Curie-Skłodowskiej w Lublinie stwierdzono występowanie 418 gatunków motyli nocnych. Lista gatunków zawiera: 116 gatunków należących do Noctuidae $(26,4 \%$ polskiej fauny), 116 gatunków należących do Geometridae (28,4\% polskiej fauny) oraz 63 gatunki należące do pozostałych przedstawicieli Macrolepidoptera (27,9\% polskiej fauny). Pozostałe 123 gatunki były reprezentowane przez Microlepidoptera. Co ciekawe, ok. 10\% spośród stwierdzonych gatunków było związanych ze środowiskami podmokłymi, co stanowi zaskakująco duży odsetek w tak zurbanizowanym obszarze jak Lublin. Porównując uzyskane 
dane z poprzednimi badaniami dotyczącymi miejskiej lepidopterofauny w Polsce, zbiorowisko motyli z Ogrodu Botanicznego w Lublinie swoim składem gatunkowym było najbardziej zbliżone do tego wykazanego z rezerwatu „Las Natoliński”, który chroni pozostałość Puszczy Mazowieckiej. Spośród stwierdzonych motyli wiele należało do gatunków rzadko i lokalnie spotykanych, m.in.: Stegania cararia, Melanthia procellata, Pasiphila chloerata, Eupithecia haworthiata, Horisme corticata, Xylomoia graminea, Polychrysia moneta. W świetle przeprowadzonych badań Ogród Botaniczny w Lublinie odznacza się dosyć wysoką bioróżnorodnością i może być uważany za refugium dla motyli w środowisku miejskim Lublina.

\section{Introduction}

Moths and butterflies (Lepidoptera) are one of the groups of the largest interest amongst insects. Studies with regard to Lepidoptera are very popular since a long time and this fact could be caused, inter alia, by their indicatory relevance. Unfortunately, our world is changing relentlessly as well as it is affected by many devastating human activities which have a crucial impact on the decreasing quantity of the habitable areas for many insects (Buszko \& Masłowski 2012; Buszko \& Nowacki 2000a: 2000b: 2002; Nowacki 2000; Clark et al. 2007; Warren \& Bourn 2011). Owing to, we are in a possession of a lot of data and publications concerning Lepidoptera. Based on those papers, it may be possible to spot that some species are retreating, dying out or moving into new areas. Thus, the urban areas and their fauna have been arousing the interest of many researchers for many years. One of the first such studies, concerning the Polish area, was the paper compiled by Firganek (1878) about Lepidoptera of Nowy Sącz. This was a driver in cognition of the urban fauna of butterflies and moths in Poland. The most examined agglomeration, in this way, is Warsaw with the array of publications as of the Kreczmer's paper (1911) about rare species of the town surroundings and many further publications (Adamczewski 1949; 1951; Patryn 1947; Slatshevsky 1911; Winiarska 1982; 2000; 2002; 2004a; 2004b; Sielezniew $\&$ Stankiewicz 2002). For that matter, the following cities in Poland were examined on the same lines. Razowski \& Palik (1969) have researched the fauna of Cracow surroundings but their efforts were mostly directed to more natural areas like the Dulowska Forest, the Niepołomicka Forest and the Ojców National Park. Śliwiński \& Marciniak (1991) have studied Heterocera of Łódź with special regard to Macrolepidoptera of urban parks. Similar studies were also carried out in the Lublin area. However, they include only selected families as Noctuidae, Nolidae and Geometridae (Napiórkowska-Kowalik \& Sekuła 2008; Sekuła \& Górska-Drabik 2008). There were also conducted studies concerning the agricultural Lepidoptera pests within the Lublin limits (Górska-Drabik 2004; Napiórkowska-Kowalik \& Górska-Drabik 2004). Obviously, interest in examination of urban insect fauna and other anthropogenic ecosystems has been shared not only in Poland. Researchers from many countries have been focusing on this question and their papers have concerned both butterflies (Bonebrake \& Cooper 2014; Giuliano et al. 2004; Lintott et al. 2014; Lizeè et al. 2011; McGeoch \& Chown 1997; Nair et al. 2014; Öckinger et al. 2009; Saarinen et al. 2005) and many other insects, especially pollinators (Ahrné et al. 2009; Eremeeva \& Sushchev 2005; Matteson \& Langellotto 2010).

Hence, came the idea of an exact examination of Lepidoptera in the Botanical Garden of Maria Curie-Skłodowska University (MCSU) in Lublin. The aims were: 1) to reveal as comprehensive as possible the moth fauna of the Botanical Garden; 2) to reference environmental requirements of the recorded species; 3 ) to compare this data with previously published data from Lublin and other Polish urban areas, and 4) to assess whether the Botanical Garden may constitute a refuge for Lepidoptera within the urban area of Lublin. 


\section{Material and methods}

The specimens were collected during the years 2012-2013 in the Botanical Garden of MCSU in Lublin. The garden is located on the fringe of the city in the northwestern part of Lublin within the administrative borders at an altitude of ca. 200 m.a.s.l. Its surface amounts to 25 ha. The area embraces some fragment of the Czechówka river valley and a system of natural loess glens. These parts are overgrown by the natural riparian forest and by the oak-hornbeam forest with numerous lindens (Tilia cordata MıLL.) and maples (Acer pseudoplatanus L. and Acer platanoides L.). Altogether with those natural elements within the considered area, there are also small artificial ponds overgrown by hygrophilous and water plants. Additionally, in the close surrounding of the garden, there are plenty of yards and the "Górki Czechowskie" area which constitutes a formerly planned nature reserve with xerothermophilous vegetation and the hornbeam forest. All in all, this gives a really high diversity of potential host and nectar plants for many species of moths.

The studies were carried out at three sites. Two of them were localised at both sides of the main glen in the central part of the garden (1. and 2. site) and the third one was located next to the river valley and artificial ponds (Fig. 1). All specimens were collected using light trap methods, both night collecting sheet (NCS) and self-catching trap (SCT). In 2012 the survey was regularly carried out from 23 of March to 23 of October during the convenient atmospheric conditions with a minimal frequency once per week. During this time 35 samples were gathered. In 2013 the studies were supported by six additional sampling days.

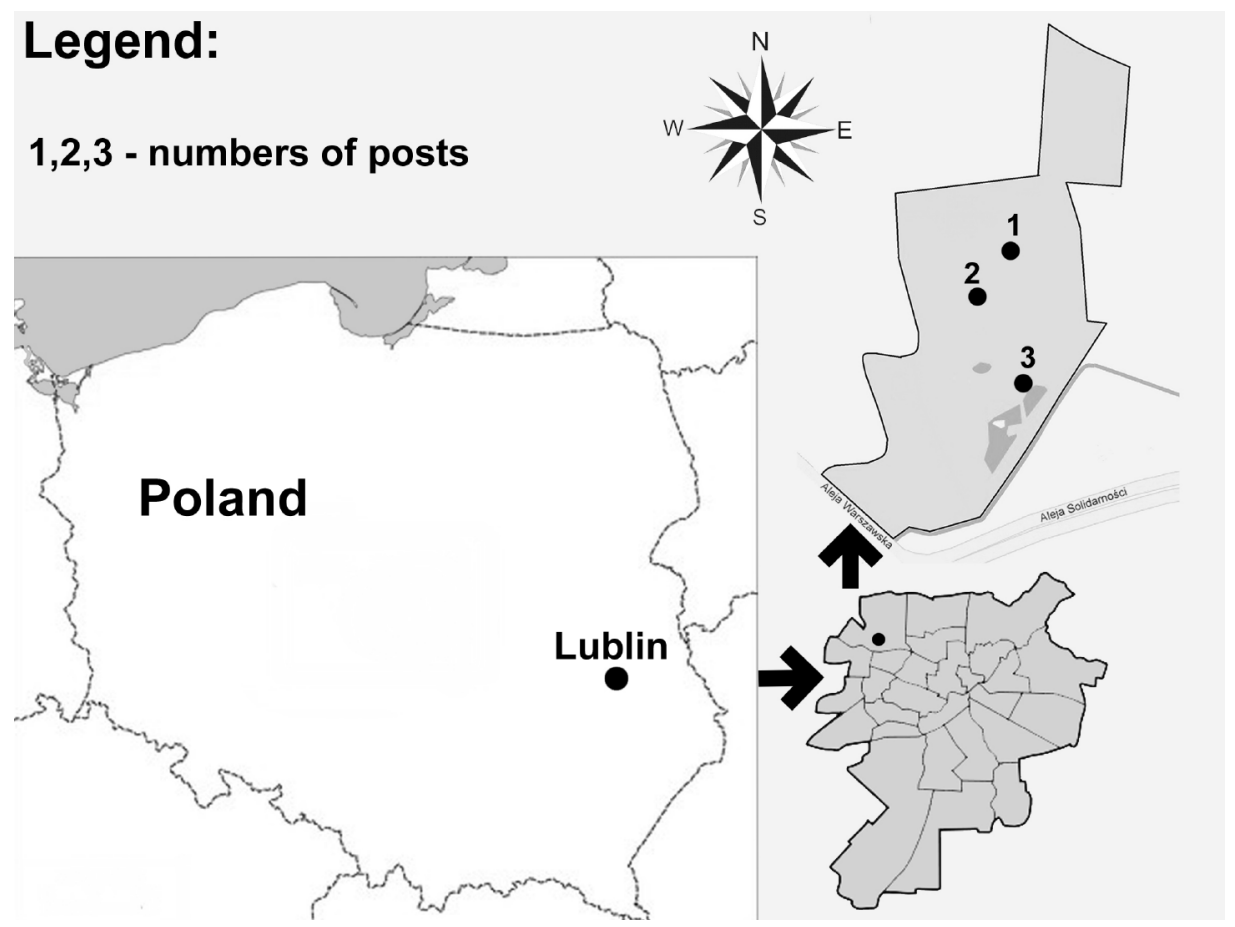

Figure 1. The research locations in the Botanical Garden of MCSU in Lublin 
Identification of moth species, classification to particular habitat preferences and rating as a rare species was followed by: Buszko (2000), Buszko \& Masłowski (2012), Malkiewicz (2012) and Nowacki (1998). In cases of vague or very worn specimens determination was carried out by genital structures examination, especially in Eupithecia Curtis, 1825 and Amphipoea Billberg, 1820 genera (Mironov, 2003; Nowacki, 1998).

The present material was analysed in order to check the number of species and species community against the previously published data about moths of Lublin and other urban areas in Poland. To analyse similarities among the species composition of Macrolepidoptera assemblages occurring in the compared regions of Poland we used the MVSP ver. 3.1. suite (UPGMA analysis and Jaccard's coefficient, Kovach 2005).

\section{Results}

The studies revealed 1584 specimens appertaining to 268 species recorded by night collecting sheet method and 2847 specimens from 316 species caught by self-catching trap method. In summary, it gives 4431 specimens belonging to 399 species of moths in 2012. The most numerously recorded species were Rivula sericealis (212 exx.), Xestia c-nigrum (185 exx.), Ochropleura plecta (174 exx.), Pleuroptya ruralis (128 exx.) and Parapoynx stratiotata (96 exx.). In total, it gives 795 specimens which constitute $17.67 \%$ out of all recorded individuals of moths in the Botanical Garden during the first year of the studies. X. c-nigrum was the most frequent species revealed by night collecting sheet, that is 42 exx. (2.65\% of all recorded moths by the method in question). On the other hand, $R$. sericealis and $X$. c-nigrum were the most numerous species recorded by self-catching trap method, i.e. 200 exx. (7.02\%) and 143 exx. (5.02\%), respectively. In addition, the surveys in 2013 revealed 19 extra species. Thus, it can be assumed that the moths checklist of the considered area is still open. Altogether, the total Heterocera checklist of the Botanical Garden, compiled on the base of the presented fieldwork, consists of 418 species (Tab. 1). Amongst them, 116 species were represented by Noctuidae, 116 species by Geometridae and 63 species by other representatives of Macrolepidoptera species, which represent 26.4\%, 28.4\% and 27.9\% of the Polish fauna, respectively. This constitutes more than was expected in such a small area like the Botanical Garden, especially taking into account the prior researches. The remaining 123 species constituted of Microlepidoptera group. The studies were predominantly focused on macromoths, hence such a low number of recorded micromoth species. From all the recorded species ca. 8.8\% were represented by species which are considered rare and local in Poland or Central Europe. Among the collected moths, there were 91 Macrolepidoptera and 120 Microlepidoptera species recorded new for the Lublin area, as well as 281 new species for the Botanical Garden (including 158 of Macrolepidoptera species). 
Table 1. The moths checklist of the Botanical Garden of MCSU in Lublin $(0-$ species previously known from the Botanical Garden; $\bullet$ - species new for the Botanical Garden, previously known from the other areas of Lublin; $\bullet$ - species new both for the Botanical Garden and Lublin area)

\begin{tabular}{|c|c|c|c|c|c|}
\hline No. & Family/ $\underline{\text { Subfamily }}$ & Remarks & No. & 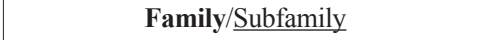 & Remarks \\
\hline \multirow[t]{3}{*}{1} & 2 & 3 & 4 & 5 & 6 \\
\hline & Noctuidae & & 20. & Heliothis viriplaca (Hufnagel, 1766) & • \\
\hline & $\underline{\text { Acontiinae }}$ & & 21. & Pyrrhia umbra (Hufnagel, 1766) & $\bullet$ \\
\hline \multirow[t]{2}{*}{1.} & Acontia trabealis (Scopoli, 1763) & $\circ$ & & $\underline{\text { Noctuinae }}$ & \\
\hline & $\underline{\text { Acronictinae }}$ & & 22. & Agrochola macilenta (Hübner, 1809) & $\bullet$ \\
\hline 2. & Acronicta alni (Linnaeus, 1767) & $\bullet$ & 23. & Agrotis exclamationis (Linnaeus, 1758) & $\circ$ \\
\hline 3. & Acronicta leporina (Linnaeus, 1758) & $\circ$ & 24. & Agrotis ipsilon (Hufnagel, 1766) & • \\
\hline 4. & Acronicta psi (Linnaeus, 1758) & $\circ$ & 25. & $\begin{array}{l}\text { Agrotis segetum (Denis \& Schiffermüller, } \\
1775 \text { ) }\end{array}$ & $\circ$ \\
\hline 5. & Acronicta rumicis (Linnaeus, 1758) & $\circ$ & 26. & Amphipoea fucosa (Freyer, 1830) & $\circ$ \\
\hline 6. & $\begin{array}{l}\text { Craniophora ligustri (Denis \& } \\
\text { Schiffermüller, 1775) }\end{array}$ & $\circ$ & 27. & $\begin{array}{l}\text { Anaplectoides prasina (Denis \& } \\
\text { Schiffermüller, 1775) }\end{array}$ & • \\
\hline 7. & Moma alpium (Osbeck, 1778) & $\bullet$ & 28. & Anarta trifolii (Hufnagel, 1766) & $\circ$ \\
\hline 8. & Simyra albovenosa (Goeze, 1781) & $\cdot$ & 29. & $\begin{array}{l}\text { Anorthoa munda (Denis \& } \\
\text { Schiffermüller, 1775) }\end{array}$ & ○ \\
\hline \multirow[t]{2}{*}{9.} & $\begin{array}{l}\text { Subacronicta megacephala (Denis \& } \\
\text { Schiffermüller, 1775) }\end{array}$ & • & 30. & Apamea crenata (Hufnagel, 1766) & $\circ$ \\
\hline & $\underline{\text { Amphipyrinae }}$ & & 31. & Apamea monoglypha (Hufnagel, 1766) & $\circ$ \\
\hline 10. & $\begin{array}{l}\text { Allophyes oxyacanthae (Linnaeus, } \\
1758 \text { ) }\end{array}$ & • & 32. & Apamea scolopacina (Esper, 1788) & $\bullet$ \\
\hline 11. & $\begin{array}{l}\text { Amphipyra livida (Denis \& } \\
\text { Schiffermüller, 1775) }\end{array}$ & $\circ$ & 33. & Apamea sordens (Hufnagel, 1766) & - \\
\hline \multirow[t]{2}{*}{12.} & $\begin{array}{l}\text { Amphipyra pyramidea (Linnaeus, } \\
1758 \text { ) }\end{array}$ & $\circ$ & 34. & Apamea unanimis (Hübner, 1813) & $\bullet$ \\
\hline & $\underline{\text { Condicinae }}$ & & 35. & $\begin{array}{l}\text { Apterogenum ypsillon (Denis \& } \\
\text { Schiffermüller, 1775) }\end{array}$ & • \\
\hline \multirow[t]{2}{*}{13.} & Eucarta virgo (Treitschke, 1835) & $\circ$ & 36. & Archanara dissoluta (Treitschke, 1825) & $\bullet$ \\
\hline & $\underline{\text { Cuculliinae }}$ & & 37. & Axylia putris (Linnaeus, 1761) & $\circ$ \\
\hline 14. & Cucullia fraudatrix Eversmann, 1837 & $\bullet$ & 38. & Caradrina morpheus (Hufnagel, 1766) & $\circ$ \\
\hline \multirow[t]{2}{*}{15.} & Cucullia umbratica (Linnaeus, 1758) & - & 39. & Caradrina selini Boisduval, 1840 & $\bullet$ \\
\hline & $\underline{\text { Dilobinae }}$ & & 40. & Charanyca ferruginea (Esper, 1785) & $\circ$ \\
\hline \multirow[t]{2}{*}{16.} & $\begin{array}{l}\text { Diloba caeruleocephala (Linnaeus, } \\
1758 \text { ) }\end{array}$ & $\bullet$ & 41. & Conistra rubiginosa (Scopoli, 1763) & $\bullet$ \\
\hline & $\underline{\text { Eustrotiinae }}$ & & 42. & Conistra vaccini (Linnaeus, 1761) & $\circ$ \\
\hline 17. & Deltote bankiana (Fabricius, 1775) & $\circ$ & 43. & $\begin{array}{l}\text { Cosmia pyralina (Denis \& } \\
\text { Schiffermüller, 1775) }\end{array}$ & ○ \\
\hline 18. & Deltote pygarga (Hufnagel, 1766) & $\circ$ & 44. & Cosmia trapezina (Linnaeus, 1758) & $\circ$ \\
\hline \multirow[t]{2}{*}{19.} & Deltote uncula (Clerck, 1759) & • & 45. & $\begin{array}{l}\text { Diarsia brunnea }(\text { Denis \& } \\
\text { Schiffermüller, 1775) }\end{array}$ & $\circ$ \\
\hline & $\underline{\text { Heliothinae }}$ & & 46. & Diarsia rubi (Vieweg, 1790) & ० \\
\hline
\end{tabular}




\begin{tabular}{|c|c|c|c|c|c|}
\hline 1 & 2 & 3 & 4 & 5 & 6 \\
\hline 47. & $\begin{array}{l}\text { Dypterygia scabriuscula (Linnaeus, } \\
1758 \text { ) }\end{array}$ & $\circ$ & 75. & Mythimna ferrago (Fabricius, 1787) & $\circ$ \\
\hline 48. & Elaphria venustula (Hübner, 1790) & $\circ$ & 76. & Mythimna impura (Hübner, 1808) & $\bullet$ \\
\hline 49. & Euplexia lucipara (Linnaeus, 1758) & $\circ$ & 77. & Mythimna l-album (Linnaeus, 1767) & $\circ$ \\
\hline 50. & Eupsilia transversa (Hufnagel, 1766) & $\circ$ & 78. & Mythimna pallens (Linnaeus, 1758) & ○ \\
\hline 51. & $\begin{array}{l}\text { Gortyna flavago (Denis \& } \\
\text { Schiffermüller, 1775) }\end{array}$ & • & 79. & $\begin{array}{l}\text { Mythimna pudorina (Denis \& } \\
\text { Schiffermüller, 1775) }\end{array}$ & $\bullet$ \\
\hline 52. & Hada plebeja (Linnaeus, 1761) & $\circ$ & 80. & Mythimna turca (Linnaeus, 1761) & ० \\
\hline 53. & Hadena bicruris (Hufnagel, 1766) & $\circ$ & 81. & $\begin{array}{l}\text { Noctua janthina (Denis \& Schiffermüller, } \\
1775 \text { ) }\end{array}$ & ○ \\
\hline 54. & $\begin{array}{l}\text { Hoplodrina ambigua (Denis \& } \\
\text { Schiffermüller, 1775) }\end{array}$ & $\circ$ & 82. & Noctua pronuba (Linnaeus, 1758) & - \\
\hline 55. & $\begin{array}{l}\text { Hoplodrina octogenaria (Goeze, } \\
1781)\end{array}$ & $\circ$ & 83. & Ochropleura plecta (Linnaeus, 1761) & ० \\
\hline 56. & Hydraecia micaea (Esper, 1789) & $\circ$ & 84. & $\begin{array}{l}\text { Oligia latruncula (Denis \& } \\
\text { Schiffermüller, 1775) }\end{array}$ & - \\
\hline 57. & Hydraecia ultima Holst, 1965 & $\circ$ & 85. & Oligia strigilis (Linnaeus, 1758) & ० \\
\hline 58. & Ipimorpha retusa (Linnaeus, 1761) & $\bullet$ & 86. & Orthosia cerasi (Fabricius, 1775) & $\circ$ \\
\hline 59. & $\begin{array}{l}\text { Ipimorpha subtusa (Denis \& } \\
\text { Schiffermüller, 1775) }\end{array}$ & $\circ$ & 87. & $\begin{array}{l}\text { Orthosia cruda (Denis \& Schiffermüller, } \\
1775 \text { ) }\end{array}$ & ० \\
\hline 60. & $\begin{array}{l}\text { Lacanobia contigua (Denis \& } \\
\text { Schiffermüller, 1775) }\end{array}$ & • & 88. & Orthosia gothica (Linnaeus, 1758) & O \\
\hline 61. & Lacanobia oleracea (Linnaeus, 1758) & ० & 89. & Orthosia incerta (Hufnagel, 1766) & ○ \\
\hline 62. & $\begin{array}{l}\text { Lacanobia suasa }(\text { Denis \& } \\
\text { Schiffermüller, 1775) }\end{array}$ & • & 90. & Orthosia populeti (Fabricius, 1775) & - \\
\hline 63. & $\begin{array}{l}\text { Lacanobia thalassina (Hufnagel, } \\
1766 \text { ) }\end{array}$ & ○ & 91. & $\begin{array}{l}\text { Phlogophora meticulosa (Linnaeus, } \\
1758 \text { ) }\end{array}$ & O \\
\hline 64. & Lacanobia w-album (Hufnagel, 1766) & • & 92. & Photedes fluxa (Hübner, 1809) & ○ \\
\hline 65. & $\begin{array}{l}\text { Lateroligia ophiogramma (Esper, } \\
\text { 1794) }\end{array}$ & $\bullet$ & 93. & Polia bombycina (Hufnagel, 1766) & ○ \\
\hline 66. & Litophane furficera (Hufnagel, 1766) & • & 94. & Polia nebeculosa (Hufnagel, 1766) & ○ \\
\hline 67. & Litophane socia (Hufnagel, 1766) & • & 95. & $\begin{array}{l}\text { Pseudeustrotia candidula (Denis \& } \\
\text { Schiffermüller, 1775) }\end{array}$ & - \\
\hline 68. & $\begin{array}{l}\text { Luperina testacea }(\text { Denis \& } \\
\text { Schiffermüller, 1775) }\end{array}$ & ○ & 96. & Rhizedra lutosa (Hübner, 1803) & ○ \\
\hline 69. & Mamestra brassicae (Linnaeus, 1758) & • & 97. & Sideridis rivularis (Fabricius, 1775) & - \\
\hline 70 . & $\begin{array}{l}\text { Melanchra persicariae (Linnaeus, } \\
\text { 1761) }\end{array}$ & ○ & 98. & Tholera decimalis (Poda, 1761) & $\circ$ \\
\hline 71. & $\begin{array}{l}\text { Mesoligia furuncula (Denis \& } \\
\text { Schiffermüller, 1775) }\end{array}$ & $\circ$ & 99. & $\begin{array}{l}\text { Tiliacea aurago (Denis \& Schiffermüller, } \\
1775 \text { ) }\end{array}$ & $\bullet$ \\
\hline 72. & $\begin{array}{l}\text { Mniotype satura }(\text { Denis \& } \\
\text { Schiffermüller, 1775) }\end{array}$ & $\bullet$ & 100. & Trachea atriplicis (Linnaeus, 1758) & ० \\
\hline 73. & $\begin{array}{l}\text { Mythimna albipuncta (Denis \& } \\
\text { Schiffermüller, 1775) }\end{array}$ & ○ & 101. & Xanthia icteritia (Hufnagel, 1766) & O \\
\hline 74. & $\begin{array}{l}\text { Mythimna conigera (Denis \& } \\
\text { Schiffermüller, 1775) }\end{array}$ & ○ & 102. & $\begin{array}{l}\text { Xestia baja (Denis \& Schiffermüller, } \\
1775 \text { ) }\end{array}$ & 0 \\
\hline
\end{tabular}




\begin{tabular}{|c|c|c|c|c|c|}
\hline 1 & 2 & 3 & 4 & 5 & 6 \\
\hline 103. & Xestia c-nigrum (Linnaeus, 1758) & ○ & 128. & Cabera exanthemata (Scopoli, 1763) & - \\
\hline 104. & $\begin{array}{l}\text { Xestia ditrapezium (Denis \& } \\
\text { Schiffermüller, 1775) }\end{array}$ & ० & 129. & Cabera pusaria (Linnaeus, 1758) & ० \\
\hline 105. & Xestia sexstrigata (Haworth, 1809) & $\bullet$ & 130. & Campaea margaritaria (Linnaeus, 1761) & $\bullet$ \\
\hline 106. & Xestia triangulum (Hufnagel, 1766) & ○ & 131. & Cephis advenaria (Hübner, 1790) & • \\
\hline 107. & $\begin{array}{l}\text { Xestia xantographa (Denis \& } \\
\text { Schiffermüller, 1775) }\end{array}$ & ○ & 132. & Chiasmia clathrata (Linnaeus, 1758) & ○ \\
\hline \multirow[t]{2}{*}{108.} & Xylomoia graminea (Graeser, 1889) & $\bullet$ & 133. & $\begin{array}{l}\text { Cleoria cinctaria (Denis \& } \\
\text { Schiffermüller, 1775) }\end{array}$ & ○ \\
\hline & Pantheinae & & 134. & Colotois pennaria (Linnaeus, 1761) & ○ \\
\hline \multirow[t]{2}{*}{109.} & Colocasia coryli (Linnaeus, 1758) & ० & 135. & $\begin{array}{l}\text { Ectropis crepuscularia (Denis \& } \\
\text { Schiffermüller, 1775) }\end{array}$ & ○ \\
\hline & $\underline{\text { Plusiinae }}$ & & 136. & Ematurga atomaria (Linnaeus, 1758) & ० \\
\hline 110. & Abrostola tripartita (Hufnagel, 1766) & ○ & 137. & Ennomos autumnaria (Werneburg, 1859) & $\circ$ \\
\hline 111. & Abrostola triplasia (Linnaeus, 1758) & $\circ$ & 138. & Ennomos fuscantaria (Haworth, 1809) & $\bullet$ \\
\hline 112. & Autographa gamma (Linnaeus, 1758) & ० & 139. & Ennomos quercinaria (Hufnagel, 1767) & ○ \\
\hline 113. & Diachrysia chrysitis (Linnaeus, 1758) & ○ & 140. & Erannis defoliaria (Clerck, 1759) & ० \\
\hline 114. & $\begin{array}{l}\text { Macdunnoughia confusa (Stephens, } \\
\text { 1850) }\end{array}$ & ○ & 141. & Hypomecis punctinalis (Scopoli, 1763) & o \\
\hline 115. & Plusia festucae (Linnaeus, 1758) & ○ & 142. & $\begin{array}{l}\text { Hypomecis roboaria (Denis \& } \\
\text { Schiffermüller, 1775) }\end{array}$ & $\circ$ \\
\hline \multirow[t]{3}{*}{116.} & Polychrysia moneta (Fabricius, 1787) & • & 143. & $\begin{array}{l}\text { Ligdia adustata (Denis \& Schiffermüller, } \\
1775 \text { ) }\end{array}$ & $\circ$ \\
\hline & Geometridae & & 144. & Lomaspilis marginata (Linnaeus, 1758) & ० \\
\hline & $\underline{\text { Alsophilinae }}$ & & 145. & $\begin{array}{l}\text { Lomographa bimaculata (Fabricius, } \\
1775 \text { ) }\end{array}$ & o \\
\hline \multirow[t]{2}{*}{117.} & $\begin{array}{l}\text { Alsophila aescularia (Denis \& } \\
\text { Schiffermüller, 1775) }\end{array}$ & ○ & 146. & $\begin{array}{l}\text { Lomographa temerata (Denis \& } \\
\text { Schiffermüller, 1775) }\end{array}$ & ० \\
\hline & $\underline{\text { Ennominae }}$ & & 147. & Lycia hirtaria (Clerck, 1759) & $\bullet$ \\
\hline 118. & Abraxas sylvata (Scopoli, 1763) & o & 148. & $\begin{array}{l}\text { Macaria alternata }(\text { Denis \& } \\
\text { Schiffermüller, 1775) }\end{array}$ & • \\
\hline 119. & Agriopis aurantiaria (Hübner, 1799) & $\cdot$ & 149. & Macaria liturata (Clerck, 1759) & ○ \\
\hline 120. & $\begin{array}{l}\text { Agriopis leucophaearia (Denis \& } \\
\text { Schiffermüller, 1775) }\end{array}$ & $\bullet$ & 150. & Macaria notata (Linnaeus, 1758) & O \\
\hline 121. & Agriopis marginaria (Fabricius, 1776) & $\cdot$ & 151. & Paradarisa consonaria (Hübner, 1799) & - \\
\hline 122. & Alcis repandata (Linnaeus, 1758) & $\circ$ & 152. & Parectropis similaria (Hufnagel, 1767) & ○ \\
\hline 123. & Apeira syringaria (Linnaeus, 1758) & • & 153. & $\begin{array}{l}\text { Phigalia pilosaria (Denis \& } \\
\text { Schiffermüller, 1775) }\end{array}$ & - \\
\hline 124. & $\begin{array}{l}\text { Ascotis selenaria }(\text { Denis \& } \\
\text { Schiffermüller, 1775) }\end{array}$ & $\bullet$ & 154. & Plagodis dolabraria (Linnaeus, 1767) & O \\
\hline 125. & Biston betularia (Linnaeus, 1758) & $\circ$ & 155. & Selenia dentaria (Fabricius, 1775) & $\circ$ \\
\hline 126. & Biston strataria (Hufnagel, 1767) & $\circ$ & 156. & Selenia tetralunaria (Hufnagel, 1767) & ० \\
\hline 127. & Bupalus piniaria (Linnaeus, 1758) & $\bullet$ & 157. & Siona lineata (Scopoli, 1763) & $\bullet$ \\
\hline
\end{tabular}




\begin{tabular}{|c|c|c|c|c|c|}
\hline 1 & 2 & 3 & 4 & 5 & 6 \\
\hline \multirow[t]{2}{*}{158.} & Stegania cararia (Hübner, 1790) & $\bullet$ & 185. & Eupithecia lariciata (Freyer, 1841) & $\bullet$ \\
\hline & Geometrinae & & 186. & Eupithecia plumbeolata (Haworth, 1809) & $\bullet$ \\
\hline \multirow[t]{2}{*}{159.} & Hemithea aestivaria (Hübner, 1789) & $\bullet$ & 187. & $\begin{array}{l}\text { Eupithecia selinata Herrich-Schäffer, } \\
1861\end{array}$ & $\bullet$ \\
\hline & $\underline{\text { Larentiinae }}$ & & 188. & Eupithecia subfuscata (Haworth, 1809) & $\circ$ \\
\hline 160. & Asthena albulata (Hufnagel, 1767) & $\bullet$ & 189. & $\begin{array}{l}\text { Eupithecia succenturiata (Linnaeus, } \\
1758 \text { ) }\end{array}$ & $\circ$ \\
\hline 161. & $\begin{array}{l}\text { Camptogramma bilineata (Linnaeus, } \\
1758 \text { ) }\end{array}$ & ○ & 190. & Eupithecia tantillaria Boisduval, 1840 & $\bullet$ \\
\hline 162. & Catorhoe cuculata (Hufnagel, 1767) & $\circ$ & 191. & $\begin{array}{l}\text { Eupithecia tripunctaria Herrich-Schäffer, } \\
1852\end{array}$ & $\bullet$ \\
\hline 163. & Chloroclystis v-ata (Haworth, 1809) & $\circ$ & 192. & Eupithecia virgaureata Doubleday, 1861 & $\bullet$ \\
\hline 164. & $\begin{array}{l}\text { Colostygia pectinataria (Knoch, } \\
1781)\end{array}$ & $\bullet$ & 193. & Eupithecia vulgata (Haworth, 1809) & • \\
\hline 165. & Cosmorhoe ocellata (Linnaeus, 1758) & $\circ$ & 194. & Horisme corticata (Treitschke, 1835) & $\bullet$ \\
\hline 166. & $\begin{array}{l}\text { Ecliptopera silaceata (Denis \& } \\
\text { Schiffermüller, 1775) }\end{array}$ & $\circ$ & 195. & Hydrelia flammeolaria (Hufnagel, 1767) & $\circ$ \\
\hline 167. & $\begin{array}{l}\text { Electrophaes corylata (Thunberg, } \\
\text { 1792) }\end{array}$ & $\circ$ & 196. & $\begin{array}{l}\text { Hydrelia sylvata (Denis \& } \\
\text { Schiffermüller, 1775) }\end{array}$ & • \\
\hline 168. & Epirrhoe alternata (Müller, 1764) & $\circ$ & 197. & Hydria cervinalis (Scopoli, 1763) & $\circ$ \\
\hline 169. & Epirrhoe rivata (Hübner, 1813) & $\bullet$ & 198. & Hydria undulata (Linnaeus, 1758) & $\circ$ \\
\hline 170. & Epirrhoe tristata (Linnaeus, 1758) & • & 199. & $\begin{array}{l}\text { Hydriomena impluviata (Denis \& } \\
\text { Schiffermüller, 1775) }\end{array}$ & • \\
\hline 171. & $\begin{array}{l}\text { Epirrita autumnata (Borkhausen, } \\
\text { 1794) }\end{array}$ & • & 200. & Lobophora halterata (Hufnagel, 1767) & • \\
\hline 172. & Eucheca nebulata (Scopoli, 1763) & $\bullet$ & 201. & $\begin{array}{l}\text { Melanthia procellata (Denis \& } \\
\text { Schiffermüller, 1775) }\end{array}$ & $\circ$ \\
\hline 173. & Eulithis mellinata (Fabricius, 1787) & $\circ$ & 202. & Operophtera brumata (Linnaeus, 1758) & • \\
\hline 174. & Eulithis prunata (Linnaeus, 1758) & ○ & 203. & Operophtera fagata (Scharfenberg, 1805) & • \\
\hline 175. & Euphyia unangulata (Haworth, 1809) & • & 204. & Orthonama vittata (Borkhausen, 1794) & • \\
\hline 176. & Eupithecia abbreviata Stephens, 1831 & $\bullet$ & 205. & Pasiphila chloerata (Mabille, 1870) & $\circ$ \\
\hline 177. & Eupithecia absinthiata (Clerck, 1759) & $\bullet$ & 206. & Pasiphila rectangulata (Linnaeus, 1758) & • \\
\hline 178. & $\begin{array}{l}\text { Eupithecia assimilata Doubleday, } \\
1856\end{array}$ & ○ & 207. & Perizoma alchemillata (Linnaeus, 1758) & $\bullet$ \\
\hline 179. & $\begin{array}{l}\text { Eupithecia centaureata (Denis \& } \\
\text { Schiffermüller, 1775) }\end{array}$ & ○ & 208. & Philereme transversata (Hufnagel, 1767) & $\bullet$ \\
\hline 180. & $\begin{array}{l}\text { Eupithecia egenaria } \text { Herrich-Schäffer, } \\
1848\end{array}$ & • & 209. & $\begin{array}{l}\text { Philereme vetulata (Denis \& } \\
\text { Schiffermüller, 1775) }\end{array}$ & $\bullet$ \\
\hline 181. & Eupithecia exiguata (Hübner, 1813) & $\bullet$ & 210. & $\begin{array}{l}\text { Plemyria rubiginata }(\text { Denis \& } \\
\text { Schiffermüller, 1775) }\end{array}$ & • \\
\hline 182. & $\begin{array}{l}\text { Eupithecia haworthiata Doubleday, } \\
1856\end{array}$ & $\bullet$ & 211. & $\begin{array}{l}\text { Pterapherapteryx sexalata (Retzius, } \\
1783 \text { ) }\end{array}$ & $\bullet$ \\
\hline 183. & Eupithecia innotata (Hufnagel, 1767) & $\circ$ & 212. & Thera juniperata (Linnaeus, 1758) & - \\
\hline 184. & $\begin{array}{l}\text { Eupithecia intricata (Zetterstedt, } \\
1839 \text { ) }\end{array}$ & • & 213. & $\begin{array}{l}\text { Thera variata (Denis \& Schiffermüller, } \\
\text { 1775) }\end{array}$ & • \\
\hline
\end{tabular}




\begin{tabular}{|c|c|c|c|c|c|}
\hline 1 & 2 & 3 & 4 & 5 & 6 \\
\hline 214. & $\begin{array}{l}\text { Xanthorhoe biriviata (Borkhausen, } \\
\text { 1794) }\end{array}$ & $\bullet$ & 238. & Cilix glaucata (Scopoli, 1763) & $\bullet$ \\
\hline 215. & $\begin{array}{l}\text { Xanthorhoe designata (Hufnagel, } \\
\text { 1767) }\end{array}$ & $\bullet$ & 239. & Drepana curvatula (Borkhausen, 1790) & $\bullet$ \\
\hline 216. & Xanthorhoe ferrugata (Clerck, 1759) & ○ & 240. & Drepana falcataria (Linnaeus, 1758) & $\bullet$ \\
\hline 217. & $\begin{array}{l}\text { Xanthorhoe fluctuata (Linnaeus, } \\
\text { 1758) }\end{array}$ & $\bullet$ & 241. & Falcaria lacertinaria (Linnaeus, 1758) & $\bullet$ \\
\hline 218. & $\begin{array}{l}\text { Xanthorhoe quadrifasiata (Clerck, } \\
1759 \text { ) }\end{array}$ & $\circ$ & 242. & Watsonalla binaria (Hufnagel, 1767) & $\bullet$ \\
\hline \multirow[t]{2}{*}{219.} & $\begin{array}{l}\text { Xanthorhoe spadicearia (Denis \& } \\
\text { Schiffermüller, 1775) }\end{array}$ & $\bullet$ & & Thyatirinae & \\
\hline & $\underline{\text { Sterrhinae }}$ & & 243. & Habrosyne pyritoides (Hufnagel, 1766) & $\bullet$ \\
\hline 220. & $\begin{array}{l}\text { Cyclophora albipunctata (Hufnagel, } \\
\text { 1767) }\end{array}$ & $\circ$ & 244. & Ochropacha duplaris (Linnaeus, 1761) & $\bullet$ \\
\hline 221. & $\begin{array}{l}\text { Cyclophora annularia (Fabricius, } \\
1775 \text { ) }\end{array}$ & $\bullet$ & 245. & Tethea or (Denis \& Schiffermüller, 1775) & $\bullet$ \\
\hline 222. & Cyclophora linearia (Hübner, 1799) & $\bullet$ & 246. & Thyatira batis (Linnaeus, 1758) & $\bullet$ \\
\hline 223. & Cyclophora pendularia (Clerck, 1759) & $\bullet$ & & Hepialidae & \\
\hline 224. & $\begin{array}{l}\text { Cyclophora punctaria (Linnaeus, } \\
1758 \text { ) }\end{array}$ & $\circ$ & 247. & Triodia sylvina (Linnaeus, 1761) & $\bullet$ \\
\hline 225. & Idaea aversata (Linnaeus, 1758) & $\circ$ & & Lasiocampidae & \\
\hline 226. & Idaea biselata (Hufnagel, 1767) & $\circ$ & & $\underline{\text { Pinarinae }}$ & \\
\hline 227. & Idaea dimidiata (Hufnagel, 1767) & $\bullet$ & 248. & Dendrolimus pini (Linnaeus, 1758) & $\bullet$ \\
\hline 228. & Scopula immorata (Linnaeus, 1758) & $\bullet$ & 249. & Odonestis pruni (Linnaeus, 1758) & $\bullet$ \\
\hline 229. & Scopula immutata (Linnaeus, 1758) & $\bullet$ & & Erebidae & \\
\hline 230. & Scopula incanata (Linnaeus, 1758) & $\bullet$ & 250. & Arctiinae & \\
\hline 231. & $\begin{array}{l}\text { Scopula nigropuctata (Hufnagel, } \\
1767 \text { ) }\end{array}$ & • & 251. & Arctia caja (Linnaeus, 1758) & $\bullet$ \\
\hline \multirow[t]{3}{*}{232.} & Timandra comae Schmidt, 1931 & ○ & 252. & Atolmis rubricollis (Linnaeus, 1758) & $\bullet$ \\
\hline & Sphingidae & & 253. & Eilema sororcula (Hufnagel, 1766) & $\bullet$ \\
\hline & Macroglossinae & & 254. & Lithosia quadra (Linnaeus, 1758) & $\bullet$ \\
\hline \multirow[t]{2}{*}{233.} & Deilephila elpenor (Linnaeus, 1758) & $\bullet$ & 255. & Pelosia muscerda (Hufnagel, 1766) & $\bullet$ \\
\hline & $\underline{\text { Smerinthinae }}$ & & 256. & $\begin{array}{l}\text { Phragmatobia fuliginosa (Linnaeus, } \\
1758 \text { ) }\end{array}$ & $\bullet$ \\
\hline 234. & Laothoe populi (Linnaeus, 1758) & $\bullet$ & 257. & Spilosoma lubricipeda (Linnaeus, 1758) & $\bullet$ \\
\hline 235. & Mimas tiliae (Linnaeus, 1758) & $\bullet$ & 258. & Spilosoma lutea (Hufnagel, 1766) & $\bullet$ \\
\hline \multirow[t]{2}{*}{236.} & Smerinthus ocellata (Linnaeus, 1758) & $\bullet$ & & $\underline{\text { Aventiinae }}$ & \\
\hline & Sphinginae & & 259. & $\begin{array}{l}\text { Laspeyria flexula (Denis \& } \\
\text { Schiffermüller, 1775) }\end{array}$ & $\circ$ \\
\hline \multirow[t]{3}{*}{237.} & Sphinx ligustri Linnaeus, 1758 & $\bullet$ & & Erebinae & \\
\hline & Drepanidae & & 259. & Catocala fulminea (Scopoli, 1763) & $\circ$ \\
\hline & Drepaninae & & 260. & Catocala nupta (Linnaeus, 1767) & $\circ$ \\
\hline
\end{tabular}




\begin{tabular}{|c|c|c|c|c|c|}
\hline 1 & 2 & 3 & 4 & 5 & 6 \\
\hline \multirow[t]{2}{*}{261.} & Lygephila pastinum (Treitschke, 1826) & ० & & $\underline{\text { Nolinae }}$ & \\
\hline & $\underline{\text { Herminiinae }}$ & & 282. & $\begin{array}{l}\text { Meganola albula (Denis \& } \\
\text { Schiffermüller, 1775) }\end{array}$ & $\bullet$ \\
\hline 262. & $\begin{array}{l}\text { Herminia grisealis (Denis \& } \\
\text { Schiffermüller, 1775) }\end{array}$ & •• & 283. & Nola aeregula (Hübner, 1793) & $\bullet$ \\
\hline 263. & Herminia tarsicrinalis (Knoch, 1782) & •• & 284. & Nola confusalis (Herrich-Schäffer, 1847) & $\circ$ \\
\hline 264. & $\begin{array}{l}\text { Herminia tarsipennalis (Treitschke, } \\
\text { 1835) }\end{array}$ & $\circ$ & & Notodontidae & \\
\hline 265. & $\begin{array}{l}\text { Macrochilo cribrumalis (Hübner, } \\
\text { 1793) }\end{array}$ & $\bullet$ & & $\underline{\text { Notodontinae }}$ & \\
\hline \multirow[t]{2}{*}{266.} & Paracolax tristalis (Fabricius, 1794) & •• & 285. & Cerura erminea (Esper, 1783) & $\bullet$ \\
\hline & $\underline{\text { Hypeninae }}$ & & 286. & Drymonia ruficornis (Hufnagel, 1766) & •• \\
\hline 267. & $\begin{array}{l}\text { Hypena proboscidalis (Linnaeus, } \\
1758 \text { ) }\end{array}$ & $\circ$ & 287. & $\begin{array}{l}\text { Leucodonta bicoloria (Denis \& } \\
\text { Schiffermüller, 1775) }\end{array}$ & $\bullet$ \\
\hline \multirow[t]{2}{*}{268.} & Hypena rostralis (Linnaeus, 1758) & $\circ$ & 288. & Notodonta dromedarius (Linnaeus, 1767) & - \\
\hline & Hypenodinae & & 289. & Notodonta ziczac (Linnaeus, 1758) & $\bullet$ \\
\hline 269. & $\begin{array}{l}\text { Hypenodes humidialis Doubleday, } \\
1850\end{array}$ & $\bullet$ & 290. & Odontosia carmelita (Esper, 1799) & $\bullet$ \\
\hline \multirow[t]{2}{*}{270.} & $\begin{array}{l}\text { Schrankia costaestrigalis (Stephens, } \\
\text { 1834) }\end{array}$ & •• & 291. & Pheosia gnoma (Fabricius, 1766) & $\bullet$ \\
\hline & Lymantriinae & & 292. & Pheosia tremulae (Clerck, 1759) & $\bullet$ \\
\hline 271. & $\begin{array}{l}\text { Calliteara pudibunda (Linnaeus, } \\
1758 \text { ) }\end{array}$ & •• & 293. & Pterostoma palpina (Clerck, 1759) & $\bullet$ \\
\hline 272. & Leucoma salicis (Linnaeus, 1758) & $\bullet$ & 294. & Ptilodon capucina (Linnaeus, 1758) & $\bullet$ \\
\hline 273. & Lymantria dispar (Linnaeus, 1758) & $\cdot$ & & Limacodidae & \\
\hline \multirow[t]{2}{*}{274.} & Lymantra monacha (Linnaeus, 1758) & •• & & $\underline{\text { Limacodinae }}$ & \\
\hline & Phytometrinae & & 295. & Apoda limacoides (Hufnagel, 1766) & •• \\
\hline 275. & $\begin{array}{l}\text { Colobochyla salicalis (Denis \& } \\
\text { Schiffermüller, 1775) }\end{array}$ & •• & & Prodoxidae & \\
\hline \multirow[t]{2}{*}{276.} & $\begin{array}{l}\text { Trisateles emortualis (Denis \& } \\
\text { Schiffermüller, 1775) }\end{array}$ & $\circ$ & 296. & Lampronia capitella (Clerck, 1759) & $\bullet$ \\
\hline & $\underline{\text { Rivulinae }}$ & & 297. & Lampronia flavimitrella (Hübner, 1817) & $\bullet$ \\
\hline \multirow[t]{2}{*}{277.} & Rivula sericealis (Scopoli, 1763) & $\circ$ & & Choreutidae & \\
\hline & $\underline{\text { Scoliopteryginae }}$ & & 298. & Anthophila fabriciana (Linnaeus, 1767) & •• \\
\hline \multirow[t]{3}{*}{278.} & Scoliopteryx libatrix (Linnaeus, 1758) & $\circ$ & & Eriocraniidae & \\
\hline & Nolidae & & 299. & $\begin{array}{l}\text { Dyseriocrania subpurpurella (Haworth, } \\
1828 \text { ) }\end{array}$ & •• \\
\hline & Chloephorinae & & & Chimabachidae & \\
\hline 279. & Earias clorana (Linnaeus, 1761) & •• & 300. & $\begin{array}{l}\text { Diurnea fagella (Denis \& Schiffermüller, } \\
\text { 1775) }\end{array}$ & $\bullet$ \\
\hline 280. & Nycteola revayana (Scopoli, 1772) & - & & Cosmopterigidae & \\
\hline 281. & $\begin{array}{l}\text { Pseudoips prasinana (Linnaeus, } \\
1758 \text { ) }\end{array}$ & $\cdot$ & & Antequerinae & \\
\hline
\end{tabular}




\begin{tabular}{|c|c|c|c|c|c|}
\hline 1 & 2 & 3 & 4 & 5 & 6 \\
\hline \multirow[t]{2}{*}{301.} & $\begin{array}{l}\text { Limnaecia phragmitella } \text { Stainton, } \\
1851\end{array}$ & $\bullet$ & 323. & $\begin{array}{l}\text { Epicallima formosella (Denis \& } \\
\text { Schiffermüller, 1775) }\end{array}$ & $\bullet$ \\
\hline & $\underline{\text { Cosmopteriginae }}$ & & 324. & Harpella forficella (Scopoli, 1763) & $\bullet$ \\
\hline 302. & $\begin{array}{l}\text { Cosmopterix orichalcea } \text { Stainton, } \\
1861\end{array}$ & $\bullet$ & 325. & Oecophora bractella (Linnaeus, 1758) & $\bullet$ \\
\hline \multirow[t]{3}{*}{303.} & $\begin{array}{l}\text { Cosmopterix zieglerella (Hübner, } \\
1810)\end{array}$ & $\bullet$ & 321. & Borkhausenia minutella (Linnaeus, 1758) & $\bullet$ \\
\hline & Elachistidae & & 322. & Crassa unitella (Hübner, 1796) & $\bullet$ \\
\hline & $\underline{\text { Depressariinae }}$ & & 323. & $\begin{array}{l}\text { Epicallima formosella (Denis \& } \\
\text { Schiffermüller, 1775) }\end{array}$ & $\bullet$ \\
\hline 304. & $\begin{array}{l}\text { Agonopterix arenella (Denis \& } \\
\text { Schiffermüller, 1775) }\end{array}$ & $\bullet$ & 324. & Harpella forficella (Scopoli, 1763) & $\bullet$ \\
\hline 305. & $\begin{array}{l}\text { Agonopterix heracliana (Linnaeus, } \\
\text { 1758) }\end{array}$ & $\bullet$ & 325. & Oecophora bractella (Linnaeus, 1758) & $\bullet$ \\
\hline 306. & $\begin{array}{l}\text { Agonopterix purpurea (Haworth, } \\
\text { 1811) }\end{array}$ & $\bullet$ & 326. & $\begin{array}{l}\text { Schiffermuelleria schaefferella } \\
\text { (Linnaeus, 1758) }\end{array}$ & $\bullet$ \\
\hline 307. & $\begin{array}{l}\text { Depressaria albipunctella (Denis \& } \\
\text { Schiffermüller, 1775) }\end{array}$ & $\bullet$ & & Bacculatricidae & \\
\hline 308. & Depressaria emeritella Stainton, 1849 & $\bullet$ & 327. & $\begin{array}{l}\text { Bucculatrix bechsteinella (Bechstein et } \\
\text { Scharfenberg, 1805) }\end{array}$ & $\bullet$ \\
\hline 309. & Depressaria chaerophylli Zeller, 1839 & $\bullet$ & 328. & Bucculatrix thoracella (Thunberg, 1794) & $\bullet$ \\
\hline 310. & Exaeretia allisella Stainton, 1849 & $\bullet$ & & Roeslerstammiidae & \\
\hline 311. & $\begin{array}{l}\text { Luquetia lobella } \text { (Denis \& } \\
\text { Schiffermüller, 1775) }\end{array}$ & $\bullet$ & 329. & $\begin{array}{l}\text { Roeslerstammia erxlebella (Fabricius, } \\
\text { 1787) }\end{array}$ & $\bullet$ \\
\hline 312. & $\begin{array}{l}\text { Orophia ferrugella (Denis \& } \\
\text { Schiffermüller, 1775) }\end{array}$ & $\bullet$ & 330. & $\begin{array}{l}\text { Roeslerstammia pronubella (Denis \& } \\
\text { Schiffermüller, 1775) }\end{array}$ & $\bullet$ \\
\hline 313. & $\begin{array}{l}\text { Semioscopis avellanella (Hübner, } \\
1793 \text { ) }\end{array}$ & $\bullet$ & & Crambidae & \\
\hline 314. & $\begin{array}{l}\text { Semioscopis oculella (Thunberg, } \\
\text { 1794) }\end{array}$ & $\bullet$ & & $\underline{\text { Acentropinae }}$ & \\
\hline \multirow[t]{2}{*}{315.} & $\begin{array}{l}\text { Semioscopis steinkellneriana (Denis } \\
\text { \& Schiffermüller, 1775) }\end{array}$ & $\bullet$ & 331. & $\begin{array}{l}\text { Acentria ephemerella (Denis \& } \\
\text { Schiffermüller, 1775) }\end{array}$ & $\bullet$ \\
\hline & $\underline{\text { Elachistinae }}$ & & 332. & Cataclysta lemnata (Linnaeus, 1758) & $\bullet$ \\
\hline 316. & Elachista argentella (Clerck, 1759) & $\bullet$ & 333. & Elophila nympheata (Linnaeus, 1758) & $\bullet$ \\
\hline \multirow[t]{2}{*}{317.} & $\begin{array}{l}\text { Elachista maculicerusella (Bruand, } \\
\text { 1859) }\end{array}$ & $\bullet$ & 334. & Parapoynx stratiotata (Linnaeus, 1758) & $\bullet$ \\
\hline & $\underline{\text { Ethmiinae }}$ & & & $\underline{\text { Crambinae }}$ & \\
\hline 318. & Ethmia bipunctella (Fabricius, 1775) & $\bullet$ & 335. & $\begin{array}{l}\text { Agriphila inquinatella (Denis \& } \\
\text { Schiffermüller, 1775) }\end{array}$ & $\bullet$ \\
\hline \multirow[t]{2}{*}{319.} & Ethmia quadrillella (Goeze, 1783) & $\bullet$ & 336. & $\begin{array}{l}\text { Agriphila straminella (Denis \& } \\
\text { Schiffermüller, 1775) }\end{array}$ & $\bullet$ \\
\hline & Oecophoridae & & 337. & Calamotropha paludella (Hübner, 1824) & $\bullet$ \\
\hline 320. & $\begin{array}{l}\text { Bisigna procerella }(\text { Denis \& } \\
\text { Schiffermüller, 1775) }\end{array}$ & $\bullet$ & 338. & $\begin{array}{l}\text { Catoptria falsella (Denis \& } \\
\text { Schiffermüller, 1775) }\end{array}$ & $\bullet$ \\
\hline 321. & $\begin{array}{l}\text { Borkhausenia minutella (Linnaeus, } \\
1758 \text { ) }\end{array}$ & $\bullet$ & 339. & $\begin{array}{l}\text { Catoptria margaritella (Denis \& } \\
\text { Schiffermüller, 1775) }\end{array}$ & $\bullet$ \\
\hline 322. & Crassa unitella (Hübner, 1796) & $\bullet$ & 340. & Catoptria verellus (Zincken, 1817) & $\bullet$ \\
\hline
\end{tabular}




\begin{tabular}{|c|c|c|c|c|c|}
\hline 1 & 2 & 3 & 4 & 5 & 6 \\
\hline 341. & Chilo phragmitella (Hübner, 1805) & •• & & $\underline{\text { Schoenobiinae }}$ & \\
\hline 342. & $\begin{array}{l}\text { Chrysoteuchia culmella (Linnaeus, } \\
1758 \text { ) }\end{array}$ & $\bullet$ & 370. & $\begin{array}{l}\text { Donacaula mucronella (Denis \& } \\
\text { Schiffermüller, 1775) }\end{array}$ & •• \\
\hline 343. & Crambus lathoniellus (Zincken, 1817) & •• & & $\underline{\text { Scopariinae }}$ & \\
\hline 344. & Crambus pascuella (Linnaeus, 1758) & .• & 371. & Eudonia pallida (Curtis, 1827) & $\bullet$ \\
\hline 345. & Crambus perlella (Scopoli, 1763) & •• & 372. & Scoparia ambigualis (Treitschke, 1829) & $\bullet$ \\
\hline 346. & Crambus uliginosellus Zeller, 1850 & •• & 373. & Scoparia basistrigalis Knaggs, 1866 & •• \\
\hline 347. & Pediasia contaminella (Hübner, 1796) & $\bullet$ & 374. & $\begin{array}{l}\text { Scoparia pyralella } \text { (Denis \& } \\
\text { Schiffermüller, 1775) }\end{array}$ & $\bullet$ \\
\hline 348. & $\begin{array}{l}\text { Pediasia luteella (Denis \& } \\
\text { Schiffermüller, 1775) }\end{array}$ & $\bullet$ & & Spilomelinae & \\
\hline \multirow[t]{2}{*}{349.} & Platytes alpinella (Hübner, 1813) & $\bullet$ & 375. & Agrotera nemoralis (Scopoli, 1763) & $\bullet$ \\
\hline & Evergestinae & & 376. & Diasema reticularis (Scopoli, 1763) & .• \\
\hline 350. & $\begin{array}{l}\text { Evergestis aenealis (Denis \& } \\
\text { Schiffermüller, 1775) }\end{array}$ & $\bullet$ & 377. & $\begin{array}{l}\text { Mecyna flavalis (Denis \& Schiffermüller, } \\
1775 \text { ) }\end{array}$ & $\bullet$ \\
\hline 351. & Evergestis extimalis (Scopoli, 1763) & $\bullet$ & 378. & $\begin{array}{l}\text { Nomophila noctuella (Denis \& } \\
\text { Schiffermüller, 1775) }\end{array}$ & $\bullet$ \\
\hline 352. & Evergetis forficalis (Linnaeus, 1758) & $\bullet$ & 379. & Pleuroptya ruralis (Scopoli, 1763) & $\bullet$ \\
\hline \multirow[t]{2}{*}{353.} & Evergetis pallidata (Hufnagel, 1767) & $\bullet$ & 380. & Udea accolalis (Zeller, 1867) & •• \\
\hline & Pyraustinae & & & Pyralidae & \\
\hline 354. & Anania coronata (Hufnagel, 1767) & •• & & $\underline{\text { Galleriinae }}$ & \\
\hline 355. & $\begin{array}{l}\text { Anania fuscalis (Denis \& } \\
\text { Schiffermüller, 1775) }\end{array}$ & $\bullet$ & 381. & Achroia grisella (Fabricius, 1794) & $\bullet$ \\
\hline 356. & Anania hortulata (Linnaeus, 1758) & $\cdot$ & 382. & Aphomia sociella (Linnaeus, 1758) & $\bullet$ \\
\hline 357. & $\begin{array}{l}\text { Anania lancealis (Denis \& } \\
\text { Schiffermüller, 1775) }\end{array}$ & •• & 383. & Aphomia zelleri Joannis, 1932 & $\bullet$ \\
\hline 358. & Anania perlucidalis (Hübner, 1809) & $\bullet$ & & Phycitinae & \\
\hline 359. & $\begin{array}{l}\text { Anania verbascalis (Denis \& } \\
\text { Schiffermüller, 1775) }\end{array}$ & $\bullet$ & 384. & Acrobasis advenella (Zincken, 1818) & $\bullet$ \\
\hline 360. & $\begin{array}{l}\text { Ecpyrrhorrhoe rubiginalis (Hübner, } \\
\text { 1796) }\end{array}$ & $\bullet$ & 385. & Assara terebrella (Zincken, 1818) & $\bullet$ \\
\hline 361. & Loxostege sticticalis (Linnaeus, 1761) & $\bullet$ & 386. & Cryptoblabes bistriga (Haworth, 1811) & $\bullet$ \\
\hline 362. & $\begin{array}{l}\text { Loxostege turbidalis (Treitschke, } \\
\text { 1829) }\end{array}$ & $\bullet$ & 387. & $\begin{array}{l}\text { Dioryctria abietella }(\text { Denis \& } \\
\text { Schiffermüller, 1775) }\end{array}$ & $\bullet$ \\
\hline 363. & Ostrinia nubilalis (Hübner, 1796) & $\bullet$ & 388. & Eccopisa effractella Zeller, 1848 & $\bullet$ \\
\hline 364. & Psammotis pulveralis (Hübner, 1796) & $\bullet$ & 389. & $\begin{array}{l}\text { Homoeosoma nebulella (Denis \& } \\
\text { Schiffermüller, 1775) }\end{array}$ & $\bullet$ \\
\hline 365. & Pyrausta aurata (Scopoli, 1763) & $\bullet$ & 390. & Moitrelia obductella (Zeller, 1839) & $\bullet$ \\
\hline 366. & Pyrausta despicata (Scopoli, 1763) & $\bullet$ & 391. & Myelois circumvoluta (Fourcroy, 1785) & $\bullet$ \\
\hline 367. & Pyrausta purpuralis (Linnaeus, 1758) & $\bullet$ & 392. & Nephopterix angustella (Hübner, 1796) & $\bullet$ \\
\hline 368. & $\begin{array}{l}\text { Sclerocona acutella (Eversmann, } \\
1842 \text { ) }\end{array}$ & 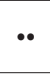 & 393. & Nyctegretis lineana (Scopoli, 1786) & $\bullet$ \\
\hline 369. & Sitochroa verticalis (Linnaeus, 1758) & $\bullet$ & 394. & Phycitodes albatella (Ragonot, 1887) & $\bullet$ \\
\hline
\end{tabular}


The Maria Curie-Skłodowska University Botanical Garden in Lublin as a refuge...

\begin{tabular}{|c|c|c|c|c|c|}
\hline 1 & 2 & 3 & 4 & 5 & 6 \\
\hline 395. & Phycitodes binavella (Hübner, 1813) & $\bullet$ & 403. & Nemaxera betulinella (Paykull, 1785) & $\bullet$ \\
\hline \multirow[t]{2}{*}{396.} & $\begin{array}{l}\text { Sciota adelphella (Fischer v. } \\
\text { Röslerstamm, 1836) }\end{array}$ & $\bullet$ & & $\underline{\text { Tineinae }}$ & \\
\hline & $\underline{\text { Pyralinae }}$ & & 404. & Monopis monachella (Hübner, 1796) & $\bullet$ \\
\hline 397. & $\begin{array}{l}\text { Endotricha flammealis (Denis \& } \\
\text { Schiffermüller, 1775) }\end{array}$ & $\bullet$ & 405. & $\begin{array}{l}\text { Monopis obviella (Denis \& } \\
\text { Schiffermüller, 1775) }\end{array}$ & $\bullet$ \\
\hline 398. & Hypsopygia costalis (Fabricius, 1775) & $\bullet$ & 406. & Tinea trinotella Thunberg, 1794 & $\bullet$ \\
\hline 395. & Phycitodes binavella (Hübner, 1813) & $\bullet$ & 407. & Tineola bisselliella (Hummel, 1823) & $\bullet$ \\
\hline \multirow[t]{2}{*}{396.} & $\begin{array}{l}\text { Sciota adelphella (Fischer v. } \\
\text { Röslerstamm, 1836) }\end{array}$ & $\bullet$ & & Argyresthiidae & \\
\hline & $\underline{\text { Pyralinae }}$ & & 408. & Argyresthia brockeella (Hübner, 1813) & $\bullet$ \\
\hline 397. & $\begin{array}{l}\text { Endotricha flammealis (Denis \& } \\
\text { Schiffermüller, 1775) }\end{array}$ & $\bullet$ & 409. & Argyresthia curvella (Linnaeus, 1761) & $\bullet$ \\
\hline 398. & Hypsopygia costalis (Fabricius, 1775) & $\bullet$ & 410. & Argyresthia goedartella (Linnaeus, 1758) & $\bullet$ \\
\hline 395. & Phycitodes binavella (Hübner, 1813) & $\bullet$ & 411. & Argyresthia trifasciata Staudinger, 1871 & $\bullet$ \\
\hline \multirow[t]{2}{*}{396.} & $\begin{array}{l}\text { Sciota adelphella (Fischer v. } \\
\text { Röslerstamm, 1836) }\end{array}$ & $\bullet$ & & Glyphipterigidae & \\
\hline & Pyralinae & & 412. & Acrolepiopsis assectella (Zeller, 1839) & $\bullet$ \\
\hline 397. & $\begin{array}{l}\text { Endotricha flammealis (Denis \& } \\
\text { Schiffermüller, 1775) }\end{array}$ & $\bullet$ & & Plutellidae & \\
\hline 398. & Hypsopygia costalis (Fabricius, 1775) & $\bullet$ & 413. & Plutella xylostella (Linnaeus, 1758) & • \\
\hline 399. & $\begin{array}{l}\text { Hypsopygia glaucinalis (Linnaeus, } \\
1758 \text { ) }\end{array}$ & $\bullet$ & 414. & Plutella porrectella (Linnaeus, 1758) & $\bullet$ \\
\hline 400. & Pyralis farinalis (Linnaeus, 1758) & $\bullet$ & & Ypsolophidae & \\
\hline \multirow[t]{3}{*}{401.} & $\begin{array}{l}\text { Pyralis regalis (Denis \& } \\
\text { Schiffermüller, 1775) }\end{array}$ & $\bullet$ & 415. & Ypsolopha asperella (Linnaeus, 1761) & $\bullet$ \\
\hline & Tineidae & & 416. & Ypsolopha dentella (Fabricius, 1775) & $\bullet$ \\
\hline & $\underline{\text { Nemapogoninae }}$ & & 417. & Ypsolopha scabrella (Linnaeus, 1761) & $\bullet$ \\
\hline 402. & $\begin{array}{l}\text { Archinemapogon yildizae Koçak, } \\
1981\end{array}$ & $\bullet$ & 418. & Ypsolopha vittella (Linnaeus, 1758) & $\bullet$ \\
\hline
\end{tabular}

The species recorded in the Botanical Garden showed diverse environmental requirements (Fig. 2). The most numerous group (190 species) was represented by species typical for deciduous forests, especially the natural oak-hornbeam forests. The second group was constituted by open-areas-liking species (141 species), characteristic for meadows, glades, gardens or ruderal areas. Another group comprised species connected with wetland sites (42 species). The thermophilous species characteristic for xerothermic grasslands and shrubs were represented by 19 recorded taxa. The species that prefer coniferous forests and other areas (dunes, heathlands, larders et al.) accounted for 12 and 14 species, respectively. 


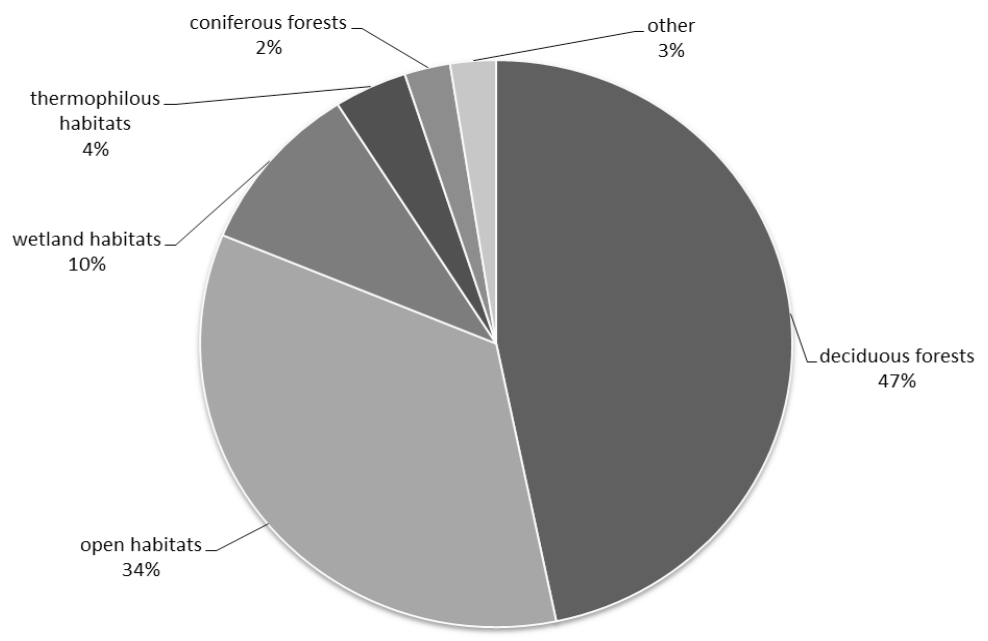

Figure 2. Habitat preferences of the recorded species

The result of UPGMA analysis was a dendrogram composed of two main branches. On one situates fauna found in the Warsaw urban area, while the second one displays faunas reported from other researched urban areas. According to the data on the moths' compositions in other, mentioned above, Polish urban areas, the species composition of moths recorded in 2012-13 in the MCSU Botanical Garden is the most similar to the fauna of the Natolin Forest Reserve (Fig. 3). The high similarity is a result of the presence of 224 common moths' species connected with deciduous forests mainly. These plant communities are predominant on both compared areas.

\section{UPGMA}

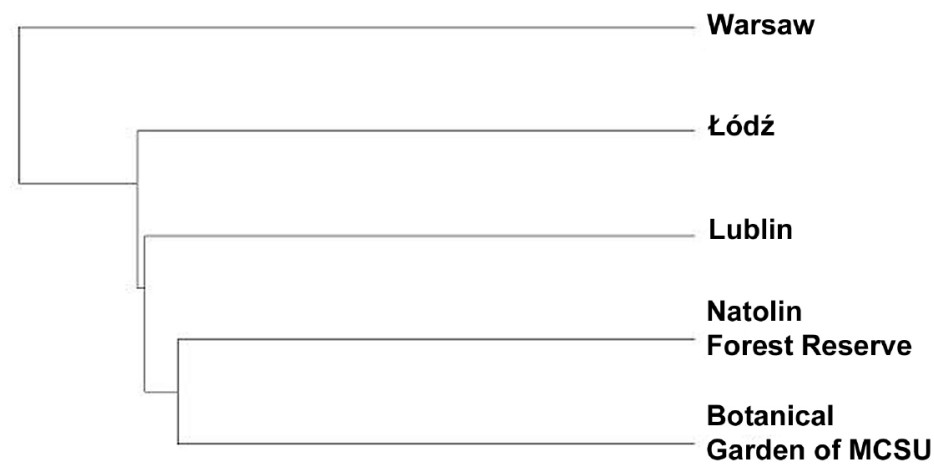

\begin{tabular}{llllllll}
\hline 0.28 & 0.4 & 0.52 & 0.64 & 0.76 & 0.88 & 1
\end{tabular}

\section{Jaccard's Coefficient}

Figure 3. The dendrogram of Macrolepidoptera assemblages similarity 


\section{Remarkable species}

About $8.8 \%$ out of all the recorded species are considered rare, have scarce distribution or are locally encountered within Poland or Central Europe. More precise information about the chosen species in question, are as follows:

Stegania cararia (Hübner, 1790)

The species is predominantly connected with riparian forests. The host plants are various poplar species (Populus L.). Distributed locally across Europe. In Poland known from scattered localities, mainly in the eastern part of the country. Rarely encountered. It reaches the north-western limit of distribution area in Poland (Buszko, 2000; Malkiewicz, 2012).

Records: 16.06.2012 - 1 ex. (SCT)

\section{Melanthia procellata (Denis \& Schiffermüller, 1775)}

The species is connected with termophilous scrubs, forest edges, parks and gardens. Caterpillars are monophags of Clematis vitalba L. Rarely encountered species, known only from scattered localities from the central, southern and western parts of Poland (Buszko, 2000; Sekuła \& Górska-Drabik, 2008).

Records: 11.05.2012 - 4 exx. (NCS), 3 exx. (SCT); 20.05.2012 - 9 exx. (NCS), 2 exx. (SCT); 03.06.2012 - 1 ex. (SCT); 16.06,2012 - 2 exx. (SCT); 06.08.2012 - 2 exx. (NCS)

Pasiphila chloerata (Mabille, 1870)

The species is characteristic for blackthorns scrubs (Prunus spinosa L.) localised on sunny slopes and forest edges. Caterpillars feed on flowers of blackthorns. Rarely encountered species, known from scattered localities from lowlands and piedmont areas (Buszko 2000).

Records: 07.06.2012 - 1 ex. (SCT); 11.06.2012 - 2 exx. (SCT); 16.06.2012 - 1 ex. (SCT)

Eupithecia selinata Herrich-Schäffer, 1861

The species is connected with deciduous and mixed forests and with clear-cut areas as well. Caterpillars feed on various species of Apiacae Lindl. Rarely encountered species, known merely from scattered localities (Buszko 2000).

Records: 16.06.2012 - 1 ex. (SCT); 24.06.2012 - 1ex. (SCT)

Eupithecia haworthiata Doubleday, 1856

The species is characteristic for thermophilous scrubs, parks and gardens. Caterpillars of this monophagous species feed on buds of Clematis vitalba L. Rarely encountered species, known only from few locations from the western, southern and south-eastern parts of Poland (Buszko et al. 1996; Buszko 2000).

Records: 16.06.2012 - 1 ex. (SCT)

Horisme corticata (Treitschke, 1835)

The species is connected with thermophilous scrubs on sunny slopes, forest edges, parks and gardens. The main host plant for this species is Clematis vitalba L., caterpillars rarely choose other congeneric species. In Poland known from scattered locations in the southern and western part of the country (Buszko 2000).

Records: $15.07 .2013-1$ ex. (NCS) 


\section{Xylomoia graminea (Graeser, 1889)}

The Asiatic species which has broadened its range recently. First record in Europe derived from 1981 from the south-western part of Russia. First recorded in Poland seven years later. This species is connected with watery areas, inter alia reeds. Its biology is poorly known, a probable host plant is Phragmites australis (Cav.) Trin. ex Steud. Rare and local species, known merely from eastern Poland. It reaches the western limit of distribution area in the country (Nowacki 1989; 1998; Bury \& Zajda 2012).

Records: 07.06.2012 - 1 ex. (SCT); 16.06.2012 - 1 ex. (SCT)

Polychrysia moneta (Fabricius, 1787)

The species prefers open areas within forests, forest-steppes, meadows and gardens. The host plants of this species belong to genera: Delphinium L., Aconitum L., Consolida Gray and Trollius L. Rarely and locally encountered species, recorded merely from a few locations in Poland. It was recently recorded in Lublin in 1968 (Napiórkowska-Kowalik \& Sekuła 2008; Nowacki 1998).

Records: 09.07.2012 - 1 ex. (NCS)

\section{Sclerocona acutella (Eversmann, 1842)}

The species prefers wetland habitats, predominantly reeds. The biology of this species is poorly known. Phragmites australis is probably its host plant. S. acutella is mostly abundant in Southern Europe. First recorded in Poland in 2011 from the Roskosz Reserve (SE Poland). It is known from majority of neighbouring countries. Probably the species has widened its distribution area lately (Buszko \& Pałka in prep.; www.entomo.pl/forum/).

Records: 11.06.2012 - 1 ex. (SCT)

\section{Udea accolalis (Zeller, 1867)}

The species is encountered within various habitats, i.a.: parks, shrublands or forest edges. Its host plants are Senecio vulgaris L. and Picris spp. L. Formerly, U. accolalis was merely known from the Subcarpathian Province (Buszko \& Nowacki 2000a). Due to the fact that the species is during expansion, it has been encountered in many locations in eastern Poland recently. Worthy a note is the fact that the species was recorded in two generations.

Records: 10.05.2012 - 4 exx. (SCT); 11.05.2012 - 7 exx. (NCS), 7 exx. (SCT); $20.05 .2012-$ 3 exx. (NCS); 03.06.2012 - 3 exx. (SCT); 09.07.2012 - 3 exx. (NCS), 6 exx. (SCT); 23.07.2012 3 exx. (SCT); 30.07.2012 - 1 ex. (SCT); 05.08.2012 - 2 exx. (SCT); 06.08.2012 - 2 exx. (NCS); 19.08.2012 - 1 ex. (SCT); 10.09.2012 -1 ex. (SCT)

\section{Discussion}

The Lepidoptera of protected regions and big urban areas are the best examined group of insects in Poland hitherto. Corresponding to the past entomological trends, many researchers merely limited to the presentation of Lepidoptera checklists.

The largest number of Macrolepidoptera species (726) has been recorded at Cracow surroundings (Razowski \& Palik 1969) so far. However, this paper predominantly focuses on natural areas outside the urbanised territory. Thus, these data are hard to compare with those described in the previously mentioned papers. On the other hand, Warsaw has the largest number of recorded species of moths within the urbanised area of the city, that is 370 of recorded moth species (but merely with 255 species after 1960 year) (Winiarska 2002; 2004a). The next, in terms of the species 
richness, are the Natolin Forest Reserve with 362 recorded species, Łódź with 333 and Lublin with 310 species (including only 163 species deriving from the Botanical Garden area) (Napiórkowska-Kowalik \& Sekuła 2008; Sekuła \& Górska-Drabik 2008; Sielezniew \& Stankiewicz 2002; Śliwiński \& Marciniak 1991). The present studies, carried out during 2012-2013, revealed 295 of Macrolepidoptera species. This constitutes almost twice as many species than prior studies of the same area.

The previous data concerning moths of Lublin derived from the whole administrative area of the city, including the Botanical Garden. These studies revealed that the most numerous moth groups were represented by species connected with forests, shrublands and open areas (i.e. ruderal habitats and meadows). In contrast, the less numerous groups were represented by thermophilous and hygrophilous species (Napiórkowska-Kowalik \& Sekuła 2008; Sekuła \& Górska-Drabik 2008), what in a large part coincides with the present data. The most numerous group (47\%) was represented by species connected with deciduous forests which was undoubtedly caused by a high share of forests in the surface of the Botanical Garden. Quite curious is the fact that the species associated with wetland areas were represented by up to $10 \%$ ( 39 species) out of all the recorded species (Fig. 2). The previous studies of the considered area has revealed merely a few and rather common species (7 species). This high share of hygrophilous moths can be obviously explained by the presence of a river valley and artificial ponds (with wealthy and next to natural vegetation) within the garden. This may be a brilliant example how artificial reservoirs and its vicinity can be utilised by many species, sometimes even rare (e.g.: Hypenodes humidalis, Schrankia costaestrigalis, X. graminea, Hydraecia ultima, Archanara dissoluta or S. acutella). The present studies confirmed the importance of urban ponds which temper the influence of progressive urbanization whereby may play an important role in maintaining a local biodiversity and protection of rare species within urbanised areas (Goertzen \& Suhling 2015; Hassall 2014).

Despite the fact that there are some differences between the Botanical Garden and the Natolin Forest Reserve in the number of recorded moths, the species composition of both are the most similar to each other amongst the rest of cited areas, what reveals the dendrogram of moths assemblages similarity (Fig. 3). It should be also pointed out that the Natolin Forest Reserve is a legacy of the old and natural Mazovian forests with an area of 110 ha wherein there is no forest management. This may suggest that such semi-artificial inside-city areas like botanical gardens can constitute the refuge for many insects, including Lepidoptera. Many authors emphasize the significance of the "green islands", including other botanical gardens, within cities and towns which play an important role in maintaining the high richness of insect assemblages in highly anthropogenic areas, e.g. Los Angeles, New York, Pretoria, Calcutta, Cape Town or McAllen (Bonebrake \& Cooper 2014; Giuliano et al. 2004; Matteson \& Langellotto 2010; McGeoch \& Chown 1997; Nair et al. 2014; Pryke \& Samways 2009; Racelis et al. 2013). Such areas can be even set beside the conservation of red listed species like in Malmö (Sweden) (Öckinger et al. 2009). Another spectacular instance about the value of urban greenery can be the case of description of 30 new to science species of Megascelia spp. (Diptera) among insect collection deriving from Los Angeles backyards (Hartop et al. 2015). In the case of Lublin, the presumption of a substantial value of this area results from high biodiversity of plants and the presence of natural environmental elements like glens, the river valley, the riparian and oak-hornbeam forest. Particularly the latter factors (that is the remnants of the natural forests) are the most valuable in the considered territory. This should be taken into account regarding any future area management. 
Lintott et al. (2014) and Racelis et al. (2013) highlight the salience of the occurrence of natural remnants or artificial plantings of native tree species which are highly beneficial for moths assemblages within the urbanised areas. This factor is in favour of the Botanical Garden to treat this place as a refuge for Lepidoptera conservation within the city limits. It is also worth mentioning that there is the "Górki Czechowskie" area in the close proximity of the garden. This is the territory of a formerly planned reserve which is characterised by high environmental diversity due to the presence of many loess glens overgrown by the oak-hornbeam forest and xerothermophilous grasslands (Balana et al. 2004). Adding to all these above-mentioned elements plenty of yards in the nearest surroundings and the fact that average annual temperatures in cities are a little higher than outside (what can make a slightly more hospitable weather condition for moths activity), all these factors can make a really convenient and habitable mixture of favourable conditions for high biodiversity. Thus, such "mixed areas" within urban limits may play a crucial role in the conservation of moth and butterfly assemblages and may be considered the hotspot within urbanised areas.

Finally, it is also worth adverting that plenty of vast structural works have been realised around the Botanical Garden after the conducted studies. That is construction of the Lublin ring road and a new housing estate in the nearest vicinity of the garden, as well as an attempt of urban development of the "Górki Czechowskie" area. Hence, the presented data could serve as an excellent data to comparable studies concerning the changes in urban habitats and their impact on the urban fauna of moths in the future.

\section{References}

Adamczewski S. 1949. Rzut oka na zmiany w faunie Warszawy i okolic wywołane przez wojnę. Polish Journal of Entomology, 18: 268-275.

Adamczewski S. 1951. Łuskoskrzydłe śródmieścia Warszawy (Lepidoptera). Fragmenta Faunistica Musei Zoologici Polonici, 6: 111-128.

Ahrné K., Bengtsson J., Elmqvist T. 2009. Bumble Bees (Bombus spp.) along a Gradient of Increasing Urbanization. PLoS ONE, 4 (5): e5574.

Balana M., Czarniawski W., Czepiel K., Gosik R., Ptaszyńska A. 2004. Walory przyrodnicze projektowanego rezerwatu Górki Czechowskie w Lublinie - stan aktualny i perspektywy. Chrońmy Przyrodę Ojczystą, 60 (1): 67-77.

Bonebrake T.C., Cooper D.S. 2014. A Hollywood drama of butterflies extirpation and persistence over a century of urbanization. Journal of Insects Conservation, 18: 683-692.

Bury J., Zajda W. 2012. Distribution of Xylomoia graminea (Graeser, 1889) (Lepidoptera: Noctuidae) in Poland. Fragmenta Faunistica, 55 (2): 139-145.

Buszko J. 2000. Atlas motyli Polski, vol. III. Falice, wycinki, miernikowce. Grupa Image, Warsaw.

Buszko J., Junnilainen J., Kaitila J., Nowacki J., Nupponen K., Pałka K. 1996. New and rare to the Polish fauna species of Lepidoptera recorded in south-eastern Poland. Entomological News, 15 (2): 105-115.

Buszko J., Masłowski J. 2012. Motyle nocne Polski, Macrolepidoptera vol. I. Wydawnictwo Koliber, Nowy Sącz.

Buszko J., Nowacki J. 2000a. The Lepidoptera of Poland, A Distributional Checklist. Polskie Towarzystwo Entomologiczne, Toruń.

Buszko J., Nowacki J. 2000b. Threat and perspectives of conservation for Lepidoptera in Poland. Entomological News, 18 Suppl. (2): 213-220. 
Buszko J., Nowacki J. 2002. Butterflies and moths (Lepidoptera). In: Red list of threatened animals in Poland. Głowaciński Z. (ed.). Instytut Ochrony Przyrody Polskiej Akademii Nauk, Cracow, pp. 80-87.

Clark P.J., Reed J.M., Chew F.S. 2007. Effects of urbanization on butterflies species richness, guild structure, and rarity. Urban Ecosystems, 10: 321-337.

Eremeeva N.I., Sushchev D.V. 2005. Structural Changes in the Fauna of Pollinating Insects in Urban Landscapes. Russian Journal of Ecology, 36 (4): 259-265.

Firganek W. 1878. Gatunki Motyli w Nowym Sączu i jego okolicy. Sprawozdania Komisji Fizjograficznej, 12.

Giuliano W.M., Accamando A.K., McAdams E.J. 2004. Lepidoptera - habitat relationships in urban parks. Urban Ecosystems, 7: 361-370.

Goertzen D., Suhling F. 2015. Central European cities maintain substantial dragonfly species richness a chance for biodiversity conservation? Insect Conservation and Diversity, 8: 238-246.

Górska-Drabik E. 2004. Motyle Lublina - cz. 2. Motyle minujące liście jabłoni w środowisku miejskim Lublina. In: Fauna miast Europy Środkowej 21. wieku. P. Indykiewicz, T. Barczak (eds.). Wydawnictwo LOGO, Bydgoszcz, pp. 119-127.

Hartop E.A., Brown B.V., Disney R.H. 2015. Opportunity in our Ignorance: Urban Biodiversity Study Reveals 30 New Species and One New Nearctic Record for Megascelia (Diptera: Phoridae) in Los Angeles (California, USA). Zootaxa, 3941 (4): 451-484.

Hassall C. 2014. The ecology and biodiversity of urban ponds. WIREs Water, 1: 187-206.

Kovach Computing Services 2005. Multi-Variate Statistical Package Plus, Version 3.1. Kovach Computing Services, Pentraeth, Wales, U.K.

Kreczmer A. 1911. Rzadkie okazy z okolic Warszawy. Entomolog Polski, 2: 17-19.

Lintott P.R., Bunnefeld N., Fuentes-Montemayor E., Minderman J., Blackmore L.M., Goulson D., Park K.J. 2014. Moths species richness, abundance and diversity in fragmented urban woodlands: implications for conservation and management strategies. Biodiversity and Conservation, 23: 2875-2901.

Lizeè M.H., Bonardo R., Mauffrey J.F., Bertaudière-Montes V., Tatoni T., Deschamps-Cottin M. 2011. Relative importance of habitat and landscape scales on butterfly communities of urbanizing areas. Comptes Rendus Biologies, 334: 74-84.

Malkiewicz A. 2012. The Geometrid Moths of Poland, vol. I. Ennominae (Lepidoptera: Geometridae). Polish Taxonomical Society, Wrocław.

Matteson K.C., Langellotto G.A. 2010. Determinates of inner city butterfly and bee species richness. Urban Ecosystems, 13: 333-347.

McGeoch M.A., Chown S.L. 1997. Impact of urbanization on a gall-inhabiting Lepidoptera assemblage: the importance of reserves in urban areas. Biodiversity and Conservation, 6: 979-993.

Mironov V. 2003. The Geometrid Moths of Europe, vol. 4: Larentinae II (Perizomini and Eupitheciini). Apollo Books, Stenstrup.

Nair A.V., Mitra P., Aditya S. 2014. Studies on the diversity and abundance of butterflies (Lepidoptera: Rhopalocera) fauna in and around Sarojini Naidu college campus, Kolkota, West Bengal, India. Journal of Entomology and Zoology Studies, 2 (4): 129-134.

Napiórkowska-Kowalik J., Górska-Drabik E. 2004. Motyle Lublina - cz. I. Ważne gospodarczo motyle (Lepidoptera) występujące w środowisku miejskim Lublina. In: Fauna miast Europy Środkowej 21. wieku. P. Indykiewicz, T. Barczak (eds.). Wydawnictwo LOGO, Bydgoszcz, pp. 103-117.

Napiórkowska-Kowalik J., Sekuła W. 2008. Motyle Lublina - cz. IV. Sówki (Lepidoptera: Noctuidae, Pantheidae, Nolidae) występujące w środowisku miejskim Lublina. In: Fauna Miast. Ochronić różnorodność biotycznq w miastach. P. Indykiewicz, L. Jerzak, T. Barczak (eds.). SAR „Pomorze”, Bydgoszcz, pp. 273-282. 
Nowacki J. 1989. Xylomoia graminea (Graeser, 1889) a noctuid moth new to the fauna of Poland and Europe (Lepidoptera, Noctuidae). Przeglad Zoologiczny, 33: 445-447.

Nowacki J. 1998. The Noctuids (Lepidoptera, Noctuidae) of Central Europe. Bratislava.

Nowacki J. 2000. Insect protection as a necessary component of nature conservation. Entomological News, 18 Supl. (2): 7-14.

Öckinger E., Dannestam Å., Smith H.G. 2009. The importance of fragmentation and habitat quality of urban grasslands for butterflies diversity. Landscape and Urban Planning, 93: 32-37.

Patryn W. 1947. Spis motyli dziennych (Rhopalocera) i nocnych (Heterocera) zebranych w okolicach Warszawy. Materiaty do Fizjografii Kraju, 5: 1-50.

Pryke J.S., Samways M.J. 2009. Recovery of invertebrate diversity in a rehabilitated city landscape mosaic in the heart of a biodiversity hotspot. Landscape and Urban Planning, 93: 54-62.

Racelis A.E., Vacek A., Goolsby C., Brush J., Goolsby J. 2013. Arthropod abundance and diversity in street trees of south Texas, USA. Subtropical Plant Science, 65: 31-37.

Razowski J., Palik E. 1969. Fauna motyli okolic Krakowa. Acta Zoologica Cracoviensia, 15: 1-309.

Saarinen K., Valtonen A., Jantuen J., Saarnio S. 2005. Butterflies and diurnal moths along road verges: Does road type affect diversity and abundance? Biological Conservation, 123: 403-412.

Sekuła W., Górska-Drabik E. 2008. Motyle Lublina - cz. V Miernikowcowate (Lepidoptera: Geometridae) występujące w środowisku miejskim Lublina. In: Fauna Miast. Ochronić różnorodność biotyczna w miastach. P. Indykiewicz, L. Jerzak, T. Barczak (eds.). SAR „Pomorze”, Bydgoszcz, pp. 283-289.

Sielezniew M., Stankiewicz A. 2002. Motyle tzw. większe (Macrolepidoptera, Heterocera) rezerwatu „Las Natoliński” w Warszawie. Parki Narodowe i Rezerwaty Przyrody, 21 (2): 195-205.

Slatshevsky P. 1911. Macrolepidopterofauna der Warschauer Gouvernements. Honorary Society Entomo Rossicae, 40: 1-132.

Śliwiński Z., Marciniak B. 1991. Zmiany w składzie gatunkowym motyli na terenie parków łódzkich w latach 1946-1987. Acta Universitatis Lodziensis Folia Zoologica et Arthropoda, 7: 131-154.

Warren M.S, Bourn N.A. 2011. Ten challenges for 2010 and beyond to conserve Lepidoptera in Europe. Journal of Insect Conservation, 15: 321-326.

Winiarska G. 1982. Noctuids (Noctuidae, Lepidoptera) of Warsaw and Mazovia. In: Species composition and origin of the fauna of Warsaw. W. Czechowski, H. Garbarczyk, B. Pisarski, J. Sawoniewicz (eds.). Memorabilia Zoologica, 36: 185-200.

Winiarska G. 2000. Insect in cities - selected problems of the threat to and protection of entomofauna in urban ecosystems. Entomological News, 18 Supl. (2): 121-128.

Winiarska G. 2002. Butterflies and moths (Lepidoptera) in urban habitats: the moths of Warsaw. I. Noctuidae, Pantheidae, Nolidae. Fragmenta Faunistica, 45: 131-145.

Winiarska G. 2004a. Butterflies and moths (Lepidoptera) in urban habitats: the moths of Warsaw. III. Noctuoidea (second part): Notodontidae, Arctiidae, Lymantriidae. Fragmenta Faunistica, 47 (2): $121-126$.

Winiarska G. 2004b. The urban moths. Academia. The Magazine of the Polish Academy of Science, 4 (4): $8-11$.

http://www.entomo.pl/forum/.

Cite as: Dawidowicz Ł., Kucharczyk H. 2016. The Maria Curie-Skłodowska University Botanical Garden in Lublin as a refuge of the moths (Lepidoptera: Heterocera) within the city. Acta Biologica, 23: 15-34. DOI: 10.18276/ab.2016.23-02. 